\title{
AXPs/SGRs: Magnetars or Quark-stars?
}

\author{
Renxin $\mathrm{Xu}$ \\ School of Physics, Peking University, Beijing 100871, China; \\ r.x.xu@pku.edu.cn
}

\begin{abstract}
The magnetar model and a solid quark star model for anomalous X-ray pulsars/soft gamma-ray repeaters (AXPs/SGRs) are discussed. Different manifestations of pulsarlike stars are speculated to be due to both their nature (e.g., mass and strain) and their nurture (ambience, and consequently the type of accretion) in the solid quark star scenario. Relevant arguments made by the author's group, including a debate on solid cold quark matter, are briefly summarized too.
\end{abstract}

Key words: pulsars, neutron stars, elementary particles

\section{Historical notes on magnetars and quark-stars}

Since the discovery of radio pulsars about 40 years ago, more kinds of pulsarlike stars have been discovered, which are distinguished by their various manifestations, i.e., accretion-driven X-ray pulsars, X-ray bursters, AXPs/SGRs (anomalous X-ray pulsars/soft gamma-ray repeaters), CCOs (central compact objects in supernova remnants), DTNs (dim thermal neutron stars), and RRATS (rotating radio transient sources). Among these, the enigmatic sources, AXPs and SGRs, share three common surprising features: (i) all the sources have long spin periods $(5-12 \mathrm{~s})$ and persistent X-ray emission with luminosities well in excess of their spin-down powers, (ii) no clear evidence for them in binaries is obtained, and (iii) they show X-ray bursts, even giant flares saturating space detectors (the typical luminosity is greater than $10^{6}$ times of the Eddington one). Four SGRs (4 confirmed, 3 candidates) and eight AXPs (8 confirmed, 1 candidate) have now been discovered (see the catalog updated online at 'http://www.physics.mcgill.ca/ pulsar/magnetar/main.html')'.

Rapid rotation was generally thought to be the only energy source for pulsar emission soon after the discovery of radio pulsars (e.g., Manchester \& Tavlor, 1977) until the discovery of accretion-powered pulsars in X-ray binaries (Pringle \& Rees, 
1972). However, AXPs/SGRs have long spin periods (thus low spindown power, their X-ray luminosities are much larger than their spindown powers) and no binary companions, which rules out spin and accretion in binary system as the power sources for the emission. It was then proposed that SGR-like bursts as well as the persistent X-ray emission could plausibly be the result of field decay of ultra-magnetic neutron stars if MHD dynamo action in the proto-stars is very effective in case that the objects spin initially at periods of $\sim 1 \mathrm{~ms}$ (Duncan \& Thompson, 1992; Thompson \& Duncan, 1993, 1995). Because of the starquakes in the crusts of normal neutron stars, a self-induction electric field is created. The strong electric field could initiate avalanches of pair creation in the magnetosphere and certainly accelerate particles, resulting in a socalled magnetar corona (Beloborodov \& Thompson, 2007), from which highenergy bursts could be observed. The power source of AXPs/SGRs is actually the magnetic energy through field-reconnection there (Woods \& Thompson, 2005). This magnetar model is very popular nowadays in the astrophysical community.

Is there any other kind of possible power source for AXPs/SGRs? This fundamental question, however, could be related to a much more elementary and very important puzzle: What is the real nature of pulsar-like stars? (That is, are they really neutron stars or quark stars? For a general review, see, e.g., Horvath, 2006; Xu, 2006a). In fact, alternative power sources were also proposed for AXPs/SGRs in a solid quark star model (Horvath, 2005; Xu et al., 2006). Surely the properties of matter at high density but low temperature have been of great interests in both theoretical and observational aspects, especially in the case of compact stars (see a discussion in $\S 4$ ). Quantum chromodynamics (QCD) is believed to be the underlying theory for the elementary strong interaction, but its strong coupling at low energy scales has troubled physicsists for a very long time. Lattice QCD simulations and effective QCD models are ways for us to study the non-perturbative effects, whereas lattice QCD seems not accessible for calculating supranuclear matter at low temperature because of the still unsolved sign problem. Therefore, physicists can only approximate the state of matter at high density and low temperature with effective models.

As for the inner constitution of pulsar-like stars, nuclear matter (related to neutron stars) is one of the speculations even from the Landau's time, but quark matter (related to quark stars) is an alternative due to the fact of QCD's asymptotic freedom (Bodmer, 1971; Witten, 1984; Farhi \& Jaffe, 1984). Recently, Jaikumar, Reddy \& Steiner (2006) even proposed a crust comprised of nuggets of strange quark matter embedded in an uniform electron background, and found that a much reduced density gradient and negligible electric field would exist in such a quark star. It is a pity that we are now not able to determine confidently which state exists in reality due to the difficulty of QCD calculations. Therefore, we have to focus on astrophysical observations in the 
research. Based on the Planck-like spectrum without atomic features and the precession properties of pulsar-like stars, a solid quark matter conjecture was suggested (Xu, 2003). Additionally, other features naturally explained within this model could possibly include sub-pulse drifting in radio emission, glitches, strong magnetic fields, birth after a successful core-collapse supernova, detection of small bolometric radii, and transient bursts of GCRT J1745-3009 (Xu et al., 1999; Zhou et al., 2004; Xu, 2005a; Yue et al., 2006a; Zhu \& Xu, 2006). Besides, the observational features of AXPs/SGRs may also reflect the nature of solid quark stars, that will be focus on in this paper. Power sources in solid quark stars include the elastic energy, the quake-released gravitational energy, and the accretion-released gravitational energy of fossil disk (Xu et al., 2006). The hard X-ray bursts of AXPs/SGRs are proposed to be the results of AISq (accretion-induced starquakes) in the model.

\section{Possible issues challenging the magnetar idea}

Conventional models for AXPs/SGRs are magnetars, a kind of neutron stars with surface magnetic fields in the range $\sim\left(10^{14}-10^{15}\right) \mathrm{G}$, especially for understanding three supergiant flares (with released energy of $\sim 10^{44-47} \mathrm{erg}$ ) observed in SGRs. The quiescent X-ray emission with luminosity $\sim 10^{34-36}$ $\mathrm{erg} / \mathrm{s}$ as well as the bursts of AXPs/SGRs are supposed to be powered by magnetic field decay. However, there are still some observational or theoretical arguments which may challenge the idea, although magnetars are really popular in the astrophysical community nowadays.

In fact, it is still debatable to estimate the magnetic fields $(\propto \sqrt{P \dot{P}})$ of AXPs/SGRs simply from their period $(P)$ and period derivative $(\dot{P})$, since AXPs/SGRs could not be braked dominantly by the magnetodipole torque. AXPs/SGRs were suggested to be normal-field pulsar-like stars which are in an accretion propeller phase (Alpar, 2001; Chatteriee. Hernquist \& Naravan, 2000). But the difficulty in the latter view point is to reproduce the irregular bursts, even the superflares. That difficulty could be overcome if bursts are proposed to be the results of starquakes of solid quark stars (Owen, 2005; $\mathrm{Xu}$ et al., 2006). In addition, some problems with the magnetar scenario may arise from recent relevant research, which are summarized as following.

(i). The superstrong fields of magnetars are supposed to be created by MHDdynamo action of rapid rotating protoneutron stars with spin period $<3 \mathrm{~ms}$. The Poynting flux and the relativistic particle ejection of such a star should power effectively the supernova remnants. Numerical simulations of supernova remnant expansion with internal magnetars did shown a faster expansion when the energy injected into the supernova remnant by magnetar spin-down is taken into account (Allen \& Horvath, 2004). Such energetic remnants are then 
expected in magnetar models, but have not been detected (Vink \& Kuiper, 2006).

(ii). Dust emission around pulsar-like stars (e.g., AXPs) was proposed to test observationally the propeller scenarios of quark stars with Spitzer or SCUBA (Xu, 2005a, 2006c). Actually a recent discovery of mid-infrared emission from a cool disk around an isolated young anomalous X-ray pulsar, 4U 0142+61, has been reported (Wang, Chakrabarty \& Kaplan, 2006) although it is still a matter of debate whether significant propeller torque of fallback matter acts on the star.

(iii). In order to understand the thermal Planck-like spectra in the conventional model of neutron star atmospheres for DTNs or CCOs, strong magnetic fields (and possibly rapid rotation) are suggested. However, a problem inherent in these efforts is $(\mathrm{Xu}, 2004)$ : How to calm down the magnetospheric activities so that no AXP/SGR-like persistent and bursting emission could be radiated? This problem exists also for young "high-field" radio pulsars since no magnetar-like emission is detected from them (Gonzalez et al., 2006). The facts could be understandable if both AXPs/SGRs and "high"-field pulsars are braked effectively by propeller torques of fossil disks, with the relative accretion rates (being indicated by the ratio of co-rotation radius to magnetospheric one) to be higher in the former than in the latter.

(iv). It is still a matter of debate whether the absorption features in SGR 180620 can be interpreted by proton or electron cyclotron resonance (Xu. Wang \& Qiao, 2003). The field is only $\sim 5 \times 10^{11} \mathrm{G}$ in the context of electron cyclotron lines.

(v). The pressure should be very anisotropic in a relativistic degenerate neutron gas in equilibrium with a background of electrons and protons when the magnetic field is stronger than the critical field $\left(\sim 10^{13} \mathrm{G}\right)$. The vanishing of the equatorial pressure of the gas might result in a transverse collapse, and a stable magnetar could then be unlikely (Martinez, Rojas \& Cuesta, 2003). Such kind of quantum-magnetically induced collapse could also be possible in quark stars with ultra strong magnetic fields $\left(>10^{\sim 19} \mathrm{G}\right.$, Martinez et al., 2005). In this sense, quark matter would tolerate much higher magnetic field than neutron matter, and no neutron star with 10 (14-15) (i.e., no magnetar) could form stably. Nevertheless, more theoretical investigations of the equilibrium of nuclear or quark matter in general relativity are necessary.

In addition, the implied transverse velocity of an AXP, XTE J1810-197, indicates that its kick velocity is completely ordinary $(<400 \mathrm{~km} / \mathrm{s})$ though the AXP manifests strikingly (Helfand et al., 2007). It might be not easy to reproduce normal kicks for neutron stars with very fast rotation (initial period $P<3 \mathrm{~ms}$ ) and much high magnetic fields $\left(B \gg 10^{13} \mathrm{G}\right)$ in the magnetar model, since the initial magnetic field and the initial spin are generally consid- 
ered to be key parameters for kicks (Lai, 2004). Considering these criticisms, we think that alternative ideas for understanding AXPs/SGRs are welcome. We note that the quark-star model could be competitive, in which both gravitational and elastic energies would power the photon and particle radiation of AXPs/SGRs.

\section{AXPs/SGRs: quark-stars with fossil disks?}

Why do pulsar-like stars behave so diversely? As noted by $\mathrm{Xu}$ (2006c), two factors could be very important for their different manifestations: environment and stellar mass. A dense environment may be responsible for leaving a remnant-nebula after a supernova and for resulting possibly in a fallback accretion (e.g., via disk) around the compact star, whereas starquakes could more probably occur in massive compact stars. Bursts, as the results of quakes, could frequently appear in an accreting solid quark star (even low-mass) because of AISq (accretion-induced starquakes). In this context, various manifestations of pulsar-like stars would be speculated to be related to quark stars with different nature and nurture (fossil disks, quakes, and masses; see Table 1). According to the calculation of Fig. 2 in $\mathrm{Xu}$ et al. (2006), more gravitational energy could be released during quakes of solid quark stars with higher masses, and we may expect that SGRs emitting giant flares could be quark stars with $\sim(1-2) M_{\odot}$.

By assuming that AXPs/SGRs and "high"-field radio pulsars could be torqued effectively by fossil disks, Yue et al. (2006b) suggest that SGR 1900+14 and AXP 1E 1048.1-5937 might be quark star with $\sim 10^{-(1-2)} M_{\odot}$ mass if all AXPs and SGRs are likely grouped together in their Fig. 1. One of the "high"-field pulsar, PSR J1846-0258 in the supernova remnant Kes 75, has only been detected in the X-ray band, with a much higher ratio of X-ray luminosity to the spindown energy loss than that found in other young radio pulsars (e.g., the Crab pulsar). The timing of PSR J1846-0258 behaves like that of radio pulsars, with a braking index of 2.65 (Livingstone et al., 2006). Though the emission luminosity is smaller than the spindown power, it is still possible that PSR J1846-0258 would be actually accretion-powered if the real magnetic moment is very low. In this case, the monopole-induced potentials drop in the open field region of PSR J1846-0258 might not be high enough (due to a much lower real magnetic field) to produce significant pair plasma for radio emission. Also the potential drop of AXPs/SGRs could be too low to initiate avalanches of $e^{ \pm}$pairs in their quiescent states. However, it is worth noting that radio activity would still be possible for AXPs/SGRs after outburst, as in the case of radio emission from XTE J1810-197 (Camilo et al., 2006), since strong pair plasma could form due to quake-induced $E_{\|}$(electric field parallel to magnetic field). In a conclusion, AXPs and "high"-field radio pulsars might 
Table 1

Speculated characters of pulsar-like stars in the quark star scenario.

\begin{tabular}{|c|c|c|c|c|}
\hline \multicolumn{2}{|c|}{ manifestation } & propeller torque & starquake & mass \\
\hline $\begin{array}{l}\text { Radio } \\
\text { pulsars }\end{array}$ & $\begin{array}{r}\text { normal } \\
\text { millisecond } \\
\text { part-time } \\
\text { "high"-field } \\
\end{array}$ & $\begin{array}{l}\text { negligible } \\
\text { negligible } \\
\text { fossil disk? } \\
\text { significant } \\
\end{array}$ & $\begin{array}{l}\text { glitches } \\
\text { microglitch* } \\
\text { glitches }\end{array}$ & $\begin{array}{l}\text { some low? } \\
\text { most low? } \\
\text { low (dying)? }\end{array}$ \\
\hline $\begin{array}{l}\text { Accretion- } \\
\text { powered }\end{array}$ & $\begin{array}{r}X \text {-ray pulsars } \\
X \text {-ray bursts } \\
\end{array}$ & $\begin{array}{l}\text { strong accretion } \\
\text { strong accretion }\end{array}$ & a frequency glitch ${ }^{\dagger}$ & \\
\hline \multicolumn{2}{|c|}{ CCOs } & significant & no-detection & low? \\
\hline \multicolumn{2}{|c|}{ DTNs } & weak accretion & no-detection & low? \\
\hline \multicolumn{2}{|c|}{ AXPs } & substantial & AISq $^{\ddagger}$, frequent $^{b}$ & \\
\hline \multicolumn{2}{|c|}{ SGRs } & substantial & AISq, more frequent & $\begin{array}{l}\sim M_{\odot} \text { for those } \\
\text { with superflares }\end{array}$ \\
\hline
\end{tabular}

* Only one microglitch with $10^{-11}$ change in fractional frequency was detected in a millisecond pulsar, PSR B1821-24 (Cognard \& Backer, 2004).

$\dagger$ Still one glitch in an accreting pulsar, KS 1947+300, has been reported during outbursts, with a fractional change in frequency of $3.7 \times 10^{-5}$ to be significantly larger than the values observed in the glitches of radio pulsars (Galloway, Morgan \& Levine, 2004).

$\ddagger$ Accretion-induced starquakes (AISq).

${ }^{b}$ Large glitches are detected from two AXPs, nearly simultaneously with burst: $1 \mathrm{E}$ $2259+586\left(\Delta \nu / \nu \sim 4 \times 10^{-6}\right)$ and CXOU J164710.2-455216 $\left(\Delta \nu / \nu=6.5 \times 10^{-5}\right)$ (Israel et al., 2007).

be in a same group, with different magnetic moment but similar appearances.

The magnetospheric electrodynamics of isolated rotation-powered pulsars is studied extensively in the literatures, including pair production, particle acceleration, and $E_{\|}$-solutions. However, the corresponding dynamics of a rotating quark star in a propeller phase, with significant accretion onto the polar cap, could be in a very different scenario. Ruderman-Sutherland-type vacuum gap (Ruderman \& Sutherland, 1975) may not exist (thus no sparking occurs) above polar caps since the hot polar cap bombarded by accreted nucleons could emit sufficient charged mesons and/or hadrons in the QCD-phase conversion process. A space charge-limited flow model (Arons \& Scharlemann, 1979) may adaptable in this case, where the mesons and hadrons are accelerated effectively by $E_{\|}$and may annihilate finally (or do by other interactions) into ultra-high energy photons and neutrinos in magnetospheres. TeVphoton emission observed could be understood in this picture, which has also been noted by many authors (e.g., Zhang et al., 2003; Link \& Burgio, 2005; Neronov \& Chernyakova, 2006). Certainly, the related dynamics needs further 
investigations in order to study the detail astrophysics there.

We note that fossil disk could affect the spindown torque in two aspects. (i) Propeller torque attributes to the MHD-coupling between accretion flow and co-rotating particles at the magnetospheric radius $\left(r_{\mathrm{m}}\right)$. (ii) Particles (accelerated by $E_{\|}$) with higher number density would be ejected (and the star spins down faster) if $r_{\mathrm{m}}$ is smaller than the light cylinder radius $\left(r_{\mathrm{lc}}\right.$; the open field line region is then determined by $r_{\mathrm{m}}$ rather by $r_{\mathrm{lc}}$ ). In this case, the potential drop in the open field lines is higher than that expected for pure rotationpowered pulsars.

Conclusive evidence for quark-stars? $\mathrm{Xu}$ (2006c) had addressed that following observations could test for the quark star (even with low mass) idea: (i) to detect dust emission around pulsar-like stars; (ii) to geometrically determine the radii of distant pulsar-like stars by advanced X-ray or UV-band facilities; (iii) to detect gravitational waves from pulsar-like stars; and (iv) to search submillisecond pulsars (especially with periods $<0.5 \mathrm{~ms}$ ). Although it should certainly be very clear evidence for quark stars if we could detect sub-millisecond pulsars by advanced radio telescope (e.g., the FAST) in the future (Xu, 2007), we note that to detect dust emission form pulsar-like stars is another way to find such evidence. The maximum elastic quadrupole deformations sustainable by normal neutron stars and solid strange quark stars might result in distinguishable maximum ellipticities (Owen, 2005), and the gravitational radiation may show the nature of pulsar-like stars. Though we shouldn't detect gravitational wave because of the low spindown powers in the second LIGO science run (Abbott et al., 2005; Owen, 2006), a result of search for known pulsars in future LIGO run might only show upper limits of masses (or radii) for the targets since the gravitational wave radiation could be mass-dependent $(\mathrm{Xu}, 2006 \mathrm{~b})$. It is also worth noting that gravitational wave emission associated with the energetic superflares of SGRs may be gathered from the LIGO data (Horvath, 2005), and that, in fact, LIGO Scientific Collaboration (2007) had put an upper limit on the gravitational wave emission, $7.7 \times 10^{46} \mathrm{erg}$, for the 92.5 Hz QPO of SGR 1806-20. In addition, it is still not sure whether the small radii derived from Planckian thermal radiation indicate the real stellar surfaces or only the hot polar caps, and it is then very necessary and important to observe confidently the emission from global surface of pulsar-like stars in, e.g., soft X-ray or UV bands. To find a binary system of low mass quark star (formed via AIC) and white dwarf could also be expected in the quark star model, which might be an effective way to search quark stars with very low masses. 


\section{Conclusions and Discussions}

One of the daunting challenges nowadays is to understand the fundamental strong interaction between quarks, especially in the low-energy limit, since the coupling is asymptotically free in the limit of high-energy. Though quark matter is predicted in QCD because of asymptotic freedom, astrophysical quark stars would be still in a low-energy region where quarks are coupled nonlinearly. In this sense, AXPs/SGRs (including other pulsar-like stars) would be ideal laboratories for us to study the non-perturbative effects of QCD. Phenomenologically, a solid state of cold quark matter was suggested, on which understanding the peculiar features of AXPs/SGRs could be based. It is suggested in this paper that the various manifestations of pulsar-like stars could imply different environments and inner structure of the sources, and that the bursts of AXPs/SGRs could be the results of accretion-induced starquakes (AISq) of quark stars in their propeller phases. A solid quark star quakes as stress energy develops due to spin-down, or cooling, or even general relativistic effect.

There exist other versions (e.g., Ouyed. Elgaroy \& Dahle, 2006a; Ouyed. Leahy \& Niebergal, 2006a, b) of quark star models for AXPs/SGRs, which may show the necessary of introducing astrophysical quark matter for understanding this kind of sources. Assuming a superconducting state, Niebergal, Ouyed \& Leahy (2006) calculated the magnetic field decay due to the expulsion of spin-induced vortices, and found that the magnetic field strengths and periods remain almost unchanged for typical parameters. Though glitches in the model of Ouyed et al. could be explained with intricate details, glitches and quasi-periodic oscillations (QPOs) during superflares might hint that the cold quark matter could be in a solid state. A simple toy-model (Glampedakis, Samuelsson \& Andersson, 2006), with the inclusion of global MHD-coupling between the elastic crust and the fluid core of a normal neutron star, was suggested to explain QPOs of SGRs, but a similar model in the solid quark star scenario is worth to make in order to understand the detail observations and to know observationally the features of solid quark matter (e.g., the shear modulus), since MHD waves in the global magnetospheres could be excited by oscillations of quark stars after their quakes. It is also very necessary to calculate the quake-induced $E_{\|}$ (electric field parallel to magnetic field) and particle acceleration in both open and closed field line regions in order to model the magnetospheric activity of AXPs/SGRs after a starquake. Two large glitches $\left(\Delta \nu / \nu \sim\left(10^{-5}-10^{-6}\right)\right)$ are detected in AXPs (Israel et al., 2007; Muno et al., 2007), and it would be very urgent to search glitches in SGRs since larger glitches could be natural in this AISq scenario.

Actually, from a viewpoint of condensed matter physics, symmetries break as energy-scale decreases so that different states of matter are in the Universe. 
Cold quark matter was supposed to be approximated by ideal Fermi gas when the interaction between quarks is neglected for matter to be asymptotically dense. But a superfluid state, in which the global U(1) symmetry is broken, was also proposed since any attractive interaction between quarks may render the Fermi surface unstable. It is further suggested that a crystalline structure (i.e., the translation symmetry is broken) may form in this color superconductivity quark matter, and that the shear modulus could be 20 to 1000 times larger than those of neutron star crusts (Mannarelli, Rajagopal \& Sharma, 2007). However, another possibility of condensation in position space could still not be rule out. Quark clusters would form in this scenario, and quark matter would be solidified at low temperature. An investigation on the initial spinevolution may show signatures of quark star solidification. Star's cooling and gravitational radiation would affect its viscosity and rotation.

From $s Q G P$ to solid quark matter? The non-linear complexity of strong interaction at low energy could lead to two hot points in recent researches: the physics of relativistic heavy ion collision and the study of pulsar-like star's structure. A key point in the quark-star model suggested for AXPs/SGRs is the conjecture that quarks would be clustered in strongly coupled quark-gluon plasma (sQGP), especially in the supranuclear matter with low temperature $(\mathrm{Xu}, 2003,2004,2005 \mathrm{a}, \mathrm{b})$ when the interaction parameter $\Gamma$ (defined by the ratio of the potential energy to the kinetic energy) is very high. Solid quark matter could be very likely in case that the thermal kinematic energy of quark clusters is much lower than the interaction energy between the clusters (i.e., $\Gamma \gg 1)$. In fact, it is recently realized $\Gamma$-value of the QGP at RHIC should be much higher than that predicted by the perturbative theory of QCD (e.g., Shuryak, 2006). Therefore, the QGP created at RHIC is also sQGP, and one may expect an even higher $\Gamma$ for cold quark matter (i.e., temperature to be much lower than $1 \mathrm{MeV}$ ). Glass-like or crystal-like solid quark matter would then be possible in case of $\mathrm{keV}$-temperature. One way to test the phenomenological interaction-models for sQGP at laboratory could be calculating the quark clusters by qMD (quark molecular dynamics), where the colored and flavored quarks could be treated as semi-classical particles interacting via a Cornell-potential (Hofmann et al., 2006),

$$
V(r)=-\frac{3}{4} \frac{\alpha_{\mathrm{s}}}{r}+\kappa r
$$

Strong interaction via this potential may favor formation of quark clusters, although the interacting potential between quark clusters could be in a different type. The experimental yield of multi-quark clusters (pentaquark, teraquark) may improve our understanding of sQGP. Recent achievements about classical sQGP are presented in Gelman et al. (2006a,b). Therefore, we expect that the idea of quark clustering in cold quark matter would probably be tested experimentally. 
The beauty of non-perturbative QCD? The uncertainty of determining the composition of pulsar-like stars depends actually and strongly on the nonperturbative nature of QCD at low-energy level, which is unfortunately not well understood. This is the real reason that we cannot make sure pulsars are neutron or quark stars according only to today's QCD calculations. Pessimistically, it might be impossible forever to know the state of supranuclear matter by pure QCD calculations; and we should then have to recognize the importance of observations and phenomenology in studying pulsar's inner structures. Investigations in such a way may discover the natural "beauty" of non-perturbative QCD. As in another but simple kind of non-perturbative dynamics, the non-linear phenomena in fluid dynamics (such as turbulence and chaos), which are surely not able to be determined by the famous NavierStokes equation, do exist in nature and are beautiful. In this sense, it is not against the rule of nature's beauty to study observationally and phenomenologically the elementary strong interaction at low energy.

Acknowledgments. I thank Jorge E. Horvath, Rachid Ouyed, and Benjamin J. Owen for their helpful suggestions and useful comments on the science as well as language of the manuscript. Two referees are also sincerely acknowledged for their constructive suggestions. This work is supported by National NSF of China $(10573002,10778611)$ and by the Key Grant Project of the Chinese Ministry of Education (305001). I would like to thank Youling Yue and other members in the pulsar group of Peking University for their stimulating discussions.

\section{References}

Abbott, B. et al. Limits on Gravitational-Wave Emission from Selected Pulsars Using LIGO Data. Phys. Rev. Lett. 94, 181103, 2005.

Allen, M. P., Horvath J. E. Influence of an Internal Magnetar on Supernova Remnant Expansion. ApJ 616, 346-356, 2004.

Alpar M. A. On Young Neutron Stars as Propellers and Accretors with Conventional Magnetic Fields. ApJ 554, 1245-1254, 2001.

Arons, J., Scharlemann, E. T. Pair formation above pulsar polar caps - Structure of the low altitude acceleration zone. ApJ 231, 854-879, 1979.

Beloborodov, A. M., Thompson, C. Corona of Magnetars. ApJ 657, 967-993, 2006.

Bodmer, A. R. Collapsed nuclei. Phys. Rev. D4, 1601-1606, 1971.

Camilo, F., Ransom, S., Halpern, J. et al. Transient pulsed radio emission from a magnetar. Nature 442, 892-895, 2006.

Chatterjee, P., Hernquist, L., Narayan, R. An Accretion Model for Anomalous X-Ray Pulsars. ApJ 534, 373-379, 2000. 
Cognard, I., Backer, D. C. A Microglitch in the Millisecond Pulsar PSR B182124 in M28. ApJ 612, L125-L127, 2004.

Duncan, R. C., Thompson, C. Formation of very strongly magnetized neutron stars - Implications for gamma-ray bursts. ApJ 392, L9-L13, 1992.

Farhi, E., Jaffe, R. L. Strange matter. Phys. Rev. D30, 2379-2390 1984.

Galloway, D. K., Morgan, E. H., Levine, A. M. A Frequency Glitch in an Accreting Pulsar. ApJ 613, 1164-1172, 2004.

Gelman, B. A., Shuryak, E. V., Zahed, I. Classical strongly coupled quarkgluon plasma. I. Model and molecular dynamics simulations. Phys. Rev. C74, 044908, 2006a.

Gelman, B. A., Shuryak, E. V., Zahed, I. Classical strongly coupled quarkgluon plasma. II. Screening and equation of state. Phys. Rev. C74, 044909, $2006 \mathrm{~b}$

Glampedakis K., Samuelsson L., Andersson N., 2006, MNRAS, 371, L74 (astro-ph/0605461)

Gonzalez, M. E., Kaspi, V. M., Camilo, F., Gaensler, B. M., Pivovaroff, M. J. PSR J1119-6127 and the X-ray Emission from High Magnetic Field Radio Pulsars. in: proceedings of "Isolated Neutron Stars: from the Interior to the Surface" (April 24-28, 2006, London, UK), eds. S. Zane, R. Turolla \& D. Page, in press (astro-ph/0610522), 2006

Helfand, D. J., Chatterjee, S., Brisken, W. F., Camilo, F., Reynolds, J., van Kerkwijk, M. H., Halpern, J. P., Ransom, S. M. VLBA measurement of the transverse velocity of the magnetar XTE J1810-197. ApJ, in press (astro-ph/0703336), 2007.

Hofmann, M., Bleicher, M., Scherer, S., Neise, L., Stöcker, H., Greiner, W. Statistical Mechanics of semi-classical colored Objects. Phys. Lett. B478, $161,2000$.

Horvath, J. Energetics of the Superflare from SGR1806-20 and a Possible Associated Gravitational Wave Burst. Mod. Phys. Lett. A20, 2799-2804, 2005.

Horvath, J. What do exotic equations of state have to offer?. in: Proceedings of Isolated Neutron Stars: from the Interior to the Surface, eds. D. Page, R. Turolla, \& S. Zane, preprint (astro-ph/0609272), 2006.

Israel, G. L., et al. The Post-Burst Awakening of the Anomalous X-ray Pulsar in Westerlund 1, ApJ, in press (astro-ph/0703684), 2007.

Jaikumar, P. Reddy, S., Steiner, A. W. Strange Star Surface: A Crust with Nuggets. Phys. Rev. Lett. 96 041101, 2006.

Lai, D. Neutron star kicks and supernova asymmetry. in: Cosmic explosions in three dimensions : asymmetries in supernovae and gamma-ray bursts. Edited by P. Hoflich, P. Kumar and J. C. Wheeler, Cambridge University Press, p.276, 2004.

LIGO Scientific Collaboration. Search for gravitational wave radiation associated with the pulsating tail of the SGR 1806-20 hyperflare of 27 December 2004 using LIGO, astro-ph/0703419, 2007.

Link, B., Burgio, F. TeV $\mu$ Neutrinos from Young Neutron Stars. Phys. Rev. 
Lett. 94, 181101, 2005.

Livingstone, M. A., Kaspi, V. M., Gotthelf, E. V., Kuiper, L. A Braking Index for the Young, High Magnetic Field, Rotation-Powered Pulsar in Kesteven 75. ApJ 647, 1286-1292, 2006.

Manchester, R. N., Taylor, J. H. Pulsars. Freeman, San Francisco, 1977.

Martinez, A. P., Rojas H. P., Cuesta H. J. M. Magnetic collapse of a neutron gas: Can magnetars indeed be formed. Eur. Phys. J., C29, 111, 2003.

Martinez, A. P., Rojas, H. P., Cuesta, H. J. M., Boligan, M., Orsaria, M. G. Quark stars and quantum-magnetically induced collapse. Int. J. Mod. Phys., D14, 1959, 2005.

Mannarelli, M., Rajagopal, K., Sharma, R. The rigidity of crystalline color superconducting quark matter. hep-ph/0702021, 2007.

Muno, M. P., et al. Title: Exciting the Magnetosphere of the Magnetar CXOU J164710.2-455216 in Westerlund 1. arXiv:0704.1630v1 [astro-ph], 2007.

Neronov, A., Chernyakova, M. Hadronic model for radio-to-TeV gamma-ray emission from PSR B1259-63. in: Proceeding of The multi messenger approach to high energy gamma ray sources (astro-ph/0610139), 2006.

Niebergal, B., Ouyed, R., Leahy, D. Magnetic Field Decay and Period Evolution of Anomalous X-Ray Pulsarsin the Context of Quark Stars. ApJ 646, L17-L20, 2006.

Ouyed, R., Elgaroy, O., Dahle, H., Keränen, P. Meissner effect and vortex dynamics in quark stars. A model for soft gamma-ray repeaters. A\&A 420, 1025-1032, 2004.

Ouyed, R., Leahy, D., Niebergal, B. Fall-back crust around a quark-nova compact remnant I: The degenerate shell case with applications to SGRs, AXPs and XDINs. preprint (astro-ph/0608536), 2006a.

Ouyed, R., Leahy, D., Niebergal, B. Fall-back crust around a quark-nova compact remnant II: The degenerate torus case with applications to AXPs. preprint (astro-ph/0611133), 2006b.

Owen, B. J. Maximum Elastic Deformations of Compact Stars with Exotic Equations of State. Phys. Rev. Lett. 95, 211101, 2005

Owen, B. J. Detectability of periodic gravitational waves by initial interferometers. Class. Quantum Grav. 23, S1-S7, 2006.

Pringle, J. E., Rees, M. J. Accretion Disc Models for Compact X-Ray Sources. A\&A 21, 1, 1972.

Ruderman, M., Sutherland, P. G. Theory of pulsars - Polar caps, sparks, and coherent microwave radiation. ApJ 196, 51-72, 1975.

Shuryak, E. V. Strongly Coupled Quark-Gluon Plasma: The Status Report. in: Proceedings of Continuous Advances in QCD (hep-ph/0608177), 2006.

Thompson, C., Duncan, R. C. Neutron star dynamos and the origins of pulsar magnetism. ApJ 408, 194-217, 1993.

Thompson, C., Duncan, R. C. The soft gamma repeaters as very strongly magnetized neutron stars - I. Radiative mechanism for outbursts. MNRAS 275, 255-300, 1995.

Vink, J., Kuiper, L. Supernova remnant energetics and magnetars: no evidence 
in favour of millisecond proto-neutron stars. MNRAS 370, L14-L18, 2006.

Wang, Z. X., Chakrabarty, D., Kaplan, D. L. A debris disk around an isolated young neutron star. Nature 440, 772-775, 2006.

Wang, Z. X., Kaplan, D. L., Chakrabarty, D. A Search for Fallback Disks in Four Young Supernova Remnants. ApJ 655, 261-268, 2007.

Witten, E. Cosmic separation of phsases. Phys. Rev. D30, 272-285, 1984.

Woods, P. M., Thompson, C. Soft gamma repeaters and anomalous X-ray pulsars: magnetar candidates. in: Compact Stellar X-ray Sources, eds. W. H. G. Lewin and M. van der Klis, Cambridge University Press, p.547-586, 2006.

Xu, R. X. Solid Quark Stars?. ApJ 596, L59-L62, 2003.

$\mathrm{Xu}$, R.X. Solid bare strange quark stars. in: Young neutron stars and their environments, IAU Symp. 218, eds. F. Camilo and B. M. Gaensler, p.299, 2004.

$\mathrm{Xu}$, R. X. 1E 1207.4-5209: a low-mass bare strange star?. MNRAS 356, 359370, 2005a.

Xu, R. X. Astrophysical Quark Matter. Chin. J. A\&A 5 (Supplement), 353358, 2005b.

Xu, R. X. Pulsars and Quark stars. Chin. J. A\&A Suppl. 2, 279C286, 2006a.

$\mathrm{Xu}, \mathrm{R}$. X. To probe into pulsar's interior through gravitational waves. Astroparticle Physics 25, 212-219, 2006b.

$\mathrm{Xu}, \mathrm{R}$. X. Low-mass bare strange stars. Advances in Space Research 37, 19921995, 2006c.

$\mathrm{Xu}, \mathrm{R}$. X. Searching for sub-millisecond pulsars: a theoretical view. in: Gravitation and Astrophysics: on the occasion of the 90th year of General Relativity (Proceedings of the VII Asia-Pacific International Conference, November 23-26, 2005, Taiwan), eds. J. M. Nester, C. M. Chen, and J. P. Hsu, World Scientific, p.159-167, 2007.

Xu, R. X., Qiao, G. J., Zhang, B. PSR 0943+10: a bare strange star?. ApJ 522, L109-L112, 1999.

Xu, R. X., Tao, D. J., Yang, Y. The superflares of soft gamma ray repeaters: giant quakes in solid quark stars?. MNRAS 373, L85-L89, 2006.

$\mathrm{Xu}$, R. X., Wang, H. G., Qiao, G. J. A note on the discovery of absorption features in 1E 1207.4-5209. Chin. Phys. Lett. 20, 314-316, 2003.

Zhang, B., Dai, Z. G., Mészáros, P., Waxman, E., Harding, A. K. High-Energy Neutrinos from Magnetars. ApJ 595, 346-351, 2003.

Zhou, A. Z., Xu, R. X., Wu, X. J., Wang, N. Quakes in solid quark stars. Astropart. Phys. 22, 73-79, 2004.

Yue, Y. L., Cui, X. H., Xu, R. X. Is PSR B0943+10 a low-mass quark star?. ApJ 649, L95-L98, 2006a.

Yue, Y. L., Zhu, W. W., Xu, R. X. "High-field" pulsars torqued by accretion disk? preprint (astro-ph/0611041), 2006b.

Zhu, W. W., Xu, R. X. GCRT J1745-3009: A freely precessing radio pulsar?. MNRAS 365, L16-L20, 2006. 